\title{
LES DEFIS DU DROIT INTERNATIONAL PUBLIC CONTEMPORAIN
}

CHALLENGESOFCONTEMPORARYPUBLICINTERNATIONAL $L A W$

Vivianny Kelly Galvão

Professeur à la Faculté de Droit de l'UNIT. Doctorat en Sciences Juridiques (UFPB). Master 2 en Droit Public (UFAL). Chercheuse au Laboratoire de Droits de l'Homme (UFAL).

E-mail: viviannygalvao@hotmail.com

Received: 2017-07-30. Accepted:2017-11-26

Résumé: Les discours purement rhétorique par les États ne seront retranchés des relations internationales, comme le prouve lorsque la participation de l'individu dans ces relations est le fait efficace. En fin de compte, il y a encore de nombreux obstacles à des préjugés politiques et juridiques. Par conséquent, le principe de la prévalence des droits de l'homme est apparue comme une véritable ligne directrice du comportement de l'État, que ce soit au niveau international, que ce soit en interne. En raison du principe de la prévalence des droits de l'homme sont nés en droit international, ce qui lui donne le statut de primate norme sur le droit interne, comme le montre. La primauté du droit international sur le droit national résulte de la force obligatoire des normes créées par les sujets DIP dans la société internationale. Cependant, la nature juridique de ce principe était au-delà des règles « typiques » du droit international, on a vu que sa pertinence normatif axé sur les besoins historiques et sociaux pour protéger les droits de l'homme soulevées à la catégorie des jus cogens. Il a été démontré que la force probante signifie l'imposition au-delà de la volonté des Etats comme le manque d'intérêt des États à l'égard des droits de l'homme reste visible à travers les nombreuses condamnations dans les tribunaux internationaux des droits, sans parler des plaintes aux organes compétents.

Mots-clés: Droit International Public - Principe de la prévalence des droits de l'hommec - Jus cogens 
Abstract: The merely rhetorical discourses uttered by the states will only be extirpated from international relations, as has been proved, when the participation of the human person in these relations is actually effected. As has been seen, there are still many obstacles to political and legal bias. To this end, the principle of the prevalence of human rights has emerged as a true guideline of state behavior, whether in the international sphere or in the domestic sphere. Because the principle of the prevalence of human rights was born in international law, this gives it the status of primacy over domestic law, as demonstrated. The primacy of international law over domestic law stems from the mandatory force of the rules created by IPD subjects in international society. Although the legal nature of this principle went beyond the «typical» norms of international law, it was found that its normative relevance driven by the historical and social need to protect human rights has elevated it to the category of ius cogens. It has been shown that co-operation means imposition beyond the will of States since the lack of interest of States in the field of human rights has been visible through numerous condemnations in the International Courts of Human Rights, not to speak of denunciations to the competent organs.

Keywords: Public International Law - Principle of the prevalence of human rights - Jus cogens

\section{INTRODUCTION}

En société, on agit conformément à la nature, aux nécessités, aux intérêts, aux passions etc. La vie humaine a besoin de se trouver sous l'impulsion d'une force motrice. Cette force mise en mouvement engendre les dynamiques sociales. Vivre en société est plus qu'un simple désir humain, étant quelque chose d'inhérent à sa condition. L'existence de l'autre est toujours témoignée en tout modèle de vie humaine (ARENDT, 2012, p. 37). La graduation des espaces sociaux est pertinente pour comprendre les complexités propres à chaque cadre relationnel. Du noyau le plus élémentaire du vivre ensemble humain (famille) au plus complexe (société internationale), les conduites considérées les plus remarquables dans ce vivre ensemble passent par quelque réglementation.

Les règles de coexistence ou les portées de conduite sont des créations culturelles, autrement dit, elles ne naissent pas des lois naturelles. Il y a plusieurs processus qui servent de base à l'adaptation sociale, comme l'étiquette, par exemple, la mode, la religion, la politique etc. Pour la présente recherche, le droit assume un rôle primordial, plus particulièrement, le droit international. Dans la ligne selon laquelle le droit produit des normes de nature impositive afin de modeler les 
dynamiques sociales et apporter des réponses aux interruptions des expectatives, le droit international agit de la même façon, mais dans un espace encore plus pluriel, en raison des différences de nature, des besoins, des intérêts et des passions en jeu.

La société internationale perçue dans les moules actuels a été classiquement construite par l'action des États, bien que 1'on ait ou voir cela depuis l'Antiquité. Ainsi, la société internationale moderne surgitelle entre États, et le droit de réguler les relations d'État est notamment européen (VALLADÂO, 1961, p. 27-28). Ici, il y a le droit international disposé selon la volonté des monarques absolutistes, et étranger aux affaires domestiques, car limité par la notion de souveraineté pleine. L'indépendance des États-Unis, suivie par celle des colonies espagnoles et portugaises en Amérique, ainsi que les Guerres Mondiales, la création et le renforcement des Nations Unies et le mouvement de décolonisation de l'Afrique constituent des faits transformateurs du contenu et de la forme du droit international.

Dans la forme, le droit international est alors codifié, migrant des sources traditionnelles coutumières vers les textes pactisés. De nouveaux défis apparaissent découlant de la positivation des normes internationales. L'absence de consensus ou l'incertitude quant au degré d'engagement dans une cause déterminée créent la distinction entre hard law et soft law. Les traités internationaux s'insèrent dans la première catégorie, puisqu'une fois ratifiés ils réclament l'observance obligatoire de la part des parties contractantes, lesquelles seront responsabilisées si elles se détournent de la conduite escomptée. Quant aux positionnements, déclarations, codes de conduite, accords amiables, connus comme soft law, ils apparaissent comme mode d'entamer les dialogues autour de thèmes qui suscitent davantage de divergences, mais qui ne sont pas régis par le pacta sunt servanda (VARGAS, 2013, p. 172).

Pour ce qui est du contenu, le traitement cruel infligé à certains groupes d'être humains par des régimes autoritaires, les assassinats en masse tels les génocides au Rwanda et dans l'ancienne Yougoslavie, ainsi que l'exploitation économique des peuples orientaux et africains, ont sucité la construction d'un nouvel axe d'équilibre du droit international : la personne humaine. Cet illuminisme juridique du XXe siècle a rayonné dans tout l'ordre international et pénétré dans les ordres nationaux, installant de nouveaux fondements dans les Constitutions des États dits démocratiques. Le processus d'humanisation du droit des minorités, au sein du discours en faveur des différences, de la mémoire et de l'identité, marque aussi le contexte actuel du droit international. Les mouvements, pacifiques ou violents, pour la reconnaissance des droits élémentaires et la participation croissante des indicateurs et des actes internationaux dans la vie des États, peuvent à première vue 
projeter une nébuleuse d'idées et un système international chaotique.

L'idée de chaos est surmontable, au fur et à mesure que s'établit une orientation claire à la raison existentielle des États et de l'ordre international lui-même. Le motif de l'existence repose sur la protection de la personne humaine. Tous les défis théoriques, législatifs, politiques, doivent être orientés par la protection et l'attribution de pouvoir à la personne humaine par (et dans) le droit des gens. Le droit international exige la primauté sur les ordres nationaux, puisqu'il est centré sur l'être humain. Face à cela, la négation de la personnalité juridique internationale de l'être humain est insoutenable, tout comme la mitigation de l'accès aux cours internationales. En outre, il faut revenir sur l'analyse critique des abstractions, tels que les droits humains, leurs fondements et caractéristiques, en quête de quelque chose de plus tangible aux réalités humaines d'aujourd'hui.

Juridiquement, la prévalence des droits humains se présente comme une norme internationale impérative, en raison de la centralité de la personne humaine, et elle impose le primat du droit international sur les normes juridiques nationales. L'expression prévalence des droits humains fait retentir la clameur historique de ceux qui désirent la paix et la réalisation des capacités humaines en situation de dignité. Cette prévalence nait au sein impératif du droit international, cherche à se consolider dans la jurisprudence internationale et incide sur les États, leur imposant les réformes essentielles.

\section{LA PRIMAUTE DU DROIT INTERNATIONAL PUBLIC}

La personnalité juridique internationale de l'être humain s'inscrit dans la perception de la communauté mondiale. Il est pertinent de rappeler la distinction entre les associations humaines qui ont pour but la conservation des volitions d'autrui, représentant des personnes ayant une vie réelle et organique (communauté), et celles qui représentent des personnes ayant une vie virtuelle et mécanique (société). Dans une société, les personnes se trouvent juxtaposées et sont indépendantes les unes des autres, alors que dans une communauté il existe un lien d'intimité et de dépendance (TONNIES, [S.d], p. 97).

$\mathrm{Au}$ sein d'une communauté, circulent des citoyens du monde qui s'identifient par l'humanité elle-même, et non plus par l'ethnie, la race, le genre, la croyance, la nationalité etc. Le projet occidental d'étendue de l'american way of life comme modèle imposé (par libre adhésion ou par agression) à tous les peuples dressait la bannière de l'universalité. Peu à peu, il s'est mis à afficher sa face violente et arbitraire, souillant les idées qui reconnaissaient en chaque être humain de la Terre un élément essentiellement égal susceptible d'être présenté comme justificatif au renforcement des droits humains. Le droit à la 
différence entre alors dans les discours officiels de groupes qui, pour une multitude de raisons, désirent rompre avec ce projet occidental.

Dans les cénacles des intellectuels et des représentants d'États, les débats entre idées universalistes et idées relativistes se popularisent. Les défenseurs du multiculturalisme cheminent anachroniquement quand ils discutent si les droits humains et le droit à la différence seraient comme l'eau et l'huile (même mélangées, elles ne se dissolvent guère l'une dans l'autre) ou si, en fait, elles feraient partie d'une même substance. Plutôt que de penser l'universel, qui a déjà des relents d'entreprise en perdition, il conviendrait de se pencher sur le commun. Ni l'universel, ni l'uniforme : le commun. La primauté des droits humains ouvre, par le biais du consensus, un espace idéal à la construction d'une communauté internationale fondée sur un noyau dur de droits humains qui, sitôt étendus, réclament des réformes urgentes, aussi bien dans l'ordre international que dans les ordres nationaux. Le premier point est de la voir comme norme première, composante d'un ordre prévalent.

\subsection{Prévalence des Droits humains à partir de la primauté du droit international public.}

Les droits humains sont en intime rapport avec le droit international, et leur prévalence renvoit, nécessairement, à la notion de primauté de l'ordonnance juridique internationale. De manière planifiée, il se trouve trois questions formelles principales qui impliquent le droit des gens : a) la première concerne l'existence du droit international, car il lui manque des éléments caractérisateurs de ce que l'on nomme le Droit ; b) la seconde s'occupe de la perception de deux ordres complètement distincts, qui ne se mêlent pas, chacun réglant des rapports propres ; c) la troisième soutient la prévalence du droit international sur le droit interne (SORTO, 2013, p. 148).

Parmi les auteurs qui nient le DIP, se trouve Hart. Selon Hart, le droit est un ensemble de règles primaires et secondaires. Celles qui sont primaires réglementent des conduites, alors que les secondaires prévoient le recours officiel à la force en cas de désobéissance. Face à ce concept, Hart se demande si le droit international possède de telles caractéristiques (HART, 1961, p. 263).

Le chapître $\mathrm{X}$ de Le Concept de Droit est consacré à la critique de l'existence du droit international, signalant le manque de législation internationale, de tribunaux dotés de juridiction compulsive et de sanctions centralement organisées. Pour l'auteur, l'absence d'institutionnalisation renvoie les États à la forme simple de structure sociale (règles primaires d'obligations). La carence de règles secondaires de changement et d'adjudication qui créent une législation 
et des tribunaux, ainsi que de règles unificatrices qui indiquent les sources, ne passe pas par la portée ou par l'applicabilité, mais découle de l'existence même de l'impérativité (HART, 1961, p. 263-266). Le problème de Hart c'est que l'existence doit, au moins, rendre une conduite obligatoire, l'idée d'obligation et de devoir étant éloignée de la « probabilité de subir une sanction ou un châtiment sous menace en cas de désobéissance ». L'obligation des règles n'est pas limitée par la menace de subir une sanction.

Il convient de souligner que l'absence d'une autorité centrale internationale - comprise comme hierarchiquement supérieure, régulatrice et ordonnatrice de la conduite de tous, sans pour autant n'être jamais régulée ni ordonnée par les autres - n'implique nullement l'inexistence d'une autorité en soi. Les normes juridiques internationales attribuent des pouvoirs à un sujet ou à un organe déterminé pour appliquer, réguler et surveiller les conduites sociales. Sans l'existence d'autorités, il est impossible d'atteindre la protection des biens publics et, dans le droit international, atteindre une protection concrète de la personne humaine. Hart utilise la notion classique de quelques éléments constitutifs de l'État pour fausser le caractère juridique des normes internationales. L'autorité est un élément formel de l'État, et la réalisation du droit postule la nécessité de celle-là. (PORRÚA PEREZ, 1999, p. 297-298).

L'autorité se manifeste concrètement dans les décisions de caractère général, créatrices de droit positif, et dans les décisions de caractère particulier prises dans chaque cas concret (PORRÚA PEREZ, 1999, p. 305). Toutefois, soutenir l'existence d'une autorité internationale centrale c'est considérer que toutes les normes de droit international seraient globalement applicables. Il est erroné de confondre le caractère international des normes avec l'application globale. Le droit international a cette identité parce que c'est un droit créé par plus d'un sujet de droit international. Il peut être exclusivement appliqué dans un cadre particulier (ex. : Pacte de San José de Costa Rica, qui incide dans le cadre de l'OEA).

En fait, il est quelque peu inutile de s'attarder davantage sur les négateurs du DIP, car les idées ici présentées dépassent la simple notion d'existence du DIP et établissent un point de départ par la suite, dans la prémisse de suprématie de l'ordre international. Cependant, la critique de Hart sert à clarifier certains points quant au caractère obligatoire du DIP, d'autant qu'il existe divers courants sur ce thème. De manière générale, la doctrine internationale présente deux visions pour ce qui est du fondement du DIP : volontariste et objectiviste. Dans la théorie de l'autolimitation (Georg Jellinek), de la volonté collective (Triepel), du consentement des nations (Hall, Oppenheim) et de la délégation du droit interne (Max Wenzel), le fondement du droit international part de 
la volonté des États. Mais dans la théorie de la norme-base (Kelsen), des droits fondamentaux des États (Grotius, Wolff, Pillet, Rivier), dans la théorie sociologique (Duguit, Georges Scelle), dans le droit naturel (Louis Le Fur), dans la théorie de la nécessité (Bentham), de la nationalité (P. Stanislaw Mancini), du pacta sunt servanda (Anzilotti), le caractère obligatoire du droit international réside dans un élément situé au-dessus des États, et c'est pourquoi ils sont objectivistes.

Les théories volontaristes se fondent sur la philosophie hegelienne de centralité de la volonté des États. Aucune ne contribue au renforcement et à la centralité de l'être humain dans l'ordre international. Des fondements trouvés hors du cadre de la volonté des États qui ne se transforment pas en obstacles à la reconnaissance de la personne humaine en tant que sujet de droit international, le pacta sunt servanda représente la force obligatoire du droit international. Ce principe général de droit puise ses racines dans la religion. Anzilotti écrit en 1902 que le fondement du droit n'est pas et ne saurait être un concept légal, car il existe un point dans le processus d'établissement d'une norme en une autre où cesse le droit. Donc, le fondement ultime du droit est davantage un concept moral qu'un principe légal. Dans l'édition de 1923 de Corso di diritto internazionale, Anzilotti adopte, avec quelques adaptations, l'idée kelsenienne du pacta sunt servanda comme fondement du DIP (GAJA, 2013, p. 127).

Anzilotti défend la norme du pacta sunt servanda comme fondement du droit international, sans s'attacher nécessairement à la théorie pure du droit de Hans Kelsen. Bien que la vision d'Anzilotti soit importante pour démontrer un fondement cohérent au DIP, il convient de souligner qu'il représente l'idée dualiste de Triepel sur le rapport entre les normes de droit international et les normes de droit interne. Le courant dualiste passe au large de la saisie de pouvoir de la personne humaine dans la société internationale, puisqu'il la laisse encerclée dans les ordres juridiques internes.

La théorie de la norme-base pour expliquer la force obligatoire du droit international a été développée par Hans Kelsen. A un certain moment, Kelsen cible le pacta sunt servanda comme Grundnorm (norme fondamentale) de la pyramide normative. La théorie kelsenienne sur le rapport entre les normes de DIP et de droit interne démontre la primauté de celle-là sur cette dernière et configure une contribution remarquable à la protection de la personne humaine et à l'effectivité des droits humains.

Il y a deux courants qui tracent les caractéristiques du rapport entre le droit international et le droit interne : le courant dualiste et le courant moniste. Les idées de Heinrich Triepel représentent la vision dualiste du rapport entre les normes de droit international et celles de droit interne. Bien qu'il n'écarte pas la possibilité que le droit 
international évolue pour reconnaître d'autres groupes situés au sein des États en tant que sujets de droit international (1966, p. 13), Triepel est incisif quand il affirme que le droit interne et le droit international forment des ordres juridiques distincts en raison des rapports qu'ils réglementent. Il incombe au droit international de régler les relations entre États ; c'est pourquoi eux seuls sont sujets de droit international sous le prisme de Triepel. Et il incombe aux normes de droit interne de régir les relations entre personnes humaines, en plus de créer le droit qui réglemente le rapport de l'État avec ses administrés (1966, p.10).

Dans la mesure où l'on se trouve en présence d'ordres séparés et totalement indépendants, le dualisme nie le conflit entre normes de droit international et normes de droit interne. Chaque droit se doit de garantir, par le truchement de ses propres sources, la réalisation des conduites escomptées. Pour ce qui est de l'assimilation du droit international par le droit interne, il y a là une bifurcation dans la doctrine dualiste (ou pluraliste). Par rapport à la technique de l'incorporation, on peut : exiger l'édition d'une loi interne distincte pour l'incorporation du traité dans l'ordre juridique national (dualisme radical), vision liée à la perception de Triepel selon laquelle il n'y a point assimilation, mais reproduction des normes juridiques internationales; ou dispenser l'absence de nécessité de cette loi spécifique, car l'incorporation se produit moyennant un iter de procédé complexe, par le biais d'une approbation du Congrès et d'une promulgation de l'Exécutif (dualisme modéré). Cela étant, selon les dualistes, ratifier un traité ne fait qu'irradier ses effets sur le plan international, étant nécessaire un acte juridique interne de reproduction ou d'assimilation pour que les normes internationales produisent des effets dans l'ordre d'État.

Dans la ligne moniste, avec primauté de l'ordre interne, se trouvent Wenzel, les frères Zorn, Decencière-Ferrandière et Korovin. Dans le monisme où il y a primauté du droit international, se trouve celle que l'on nomme École de Vienne (Hans Kelsen, Alfred Verdoss, Kunz), aux côtés d'auteurs tels que Duguit, Politis, Mestre et Mosler.

La doctrine moniste soutient qu'il existe un rapport mutuel entre les deux systèmes. Ce sont deux complexes de normes de type dynamique, comme l'ordonnance juridique internationale et une ordonnance juridique d'État. Ils forment un système unitaire, mais l'une de ces ordonnances se présente subordonnée à l'autre, parce que l'une contient une norme qui détermine la production des normes de l'autre et, conséquemment, celle-ci trouve en celle-là son fondement de validité. Dans le monisme, immédiatement après la ratification ou l'adhésion, les traités irradient leurs effets juridiques sur le plan international et national, concomitamment. Il se produit une incorporation automatique des normes venues des traités dans l'ordre d'État.

Les monistes ne nient pas la possibilité de conflit entre les 
normes juridiques de droit international et celles de droit interne. De cette situation émergent deux solutions : monisme avec primauté du droit international et monisme avec primauté du droit interne. Ce dernier manifeste une forte influence de la notion hegelienne d'État caractérisé par la souveraineté absolue. L'autolimitation de l'État réduit le droit international à une représentation externe du droit d'État. Le droit international reste au service des États (JELLINEK, 2000, p. 354). Ce courant est également adopté par Georges Burdeau qui souligne la valeur juridique des déclarations de droit comme étant des énoncés de droit positif, mais restreint sa possibilité d'imposition (dans le sens de création de droit) à l'activité de l'autorité législative interne (BURDEAU, 1961, p. 21-22).

Dans le dualisme, l'être humain n'est qu'un objet de réglementation du droit international. Dans le monisme avec primauté du droit interne, il y un droit international dépendant du droit interne, réfuté comme droit autonome, un droit d'État, simplement. La critique de ces visions doit être claire. Aucune des deux n'est capable de donner de l'impulsion à l'évolution du droit international en tant qu'espace d'action de la personne humaine dans la société internationale en qualité de sujet. Toutes deux rendent non-viable dans la pratique ce que les droits humains ont déjà reconnu : la centralité de l'être humain dans l'ordre international.

On trouve un refuge dans le monisme avec primauté du droit international. En cas de conflit entre normes internes et normes internationales, celles de droit international doivent prévaloir. Son principal défenseur est Hans Kelsen. Pour lui, le droit international définit ce qu'est un État dans le sens juridique international, règle sa conduite et détermine le domaine territorial de cet État, autrement dit, la sphère de validité spaciale de l'ordre juridique d'État, sous laquelle il peut apparaître revêtu de sa qualité d'appareil de coercition. L'ordre juridique interne ne pourra établir d'actes de coercition spécifiques que pour l'espace de validité qui lui est juridico-internationalement réservé ; ces actes de coercition peuvent ainsi juste être d'État sans offense au droit international. Selon ce doctrinaire, ce n'est qu'ainsi qu'est juridiquement possible la coexistence dans l'espace de pluralité d'États, c'est-à-dire, de pluralité d'ordres coercitifs.

\section{[...] si l'on part du Droit international comme un ordre juridique valable, le concept d'Etat ne peut être défini sans référence au Droit international. Sous cet angle, il est un ordre juridique partiel, immédiat en face du droit international, relativement centralisé, ayant un domaine de validité territoriale et temporelle juridico-internationalement limité et,}


relativement à la sphère de validité matérielle, ayant une prétention à la totalité (Totalitätsanspruch) à peine limité par la réserve du Droit international (KELSEN, 1999, p. 239).

Il s'agit de la thèse qui résulte de la pyramide de normes exposée dans la Théorie Pure du Droit par Hans Kelsen. Le droit international et le droit interne forment une unité logique, il est donc impossible, du point de vue purement juridique, que deux systèmes juridiques aient une validité côte à côte (TRIEPEL, 1966, p. 18). Il n'existe pas de frontières entre le droit international et le droit interne, et les différences sont relatives, car le processus stipulé par chaque droit de création et d'exécution possède un degré de centralisation et décentralisation moindre ou majeur (CÂMARA FILHO, 1949, p. 90-91).

Le droit international détermine le cadre de validité et la raison de validité des ordres juridiques nationales. La norme de base du droit international sera aussi la dernière raison de validité des ordres juridiques nationales (KELSEN, 1995, p. 437). À la recherche de ce fondement, Kelsen affirme que les sentences prononcées par une Cour Internationale (normes ayant davantage de base dans la hierarchie de l'ordre international) se basent sur les traités qui ont créé la Cour Internationale ; quant aux traités, ils sont fondés sur la norme générale qui oblige tout État à agir en accord avec les traités célébrés : pacta sunt servanda. Le droit international coutumier se développe sur cette base et forme la première étape de l'ordre juridique international. Ensuite, a lieu la formation d'accords internationaux et, dans un troisième moment, apparaissent les normes créées par les organes établis par les traités internationaux (1995, p. 439-440).

La marque de la primauté du droit international réside dans le fait qu'il reste en elle tout le fondement d'existence et de validité des ordres d'État ${ }^{1}$, compte tenu que les normes juridiques internationales peuvent réglementer n'importe quelle matière, donc également celles qui sont réglementées par le droit interne (KELSEN, 2000, p. 498). La critique de Hart consiste dans l'affirmation kelsenienne de trouver des

\footnotetext{
1 Exemple: pour être reconnu en tant que sujet de droit, l'État doit remplir le support de fait prévu hypothétiquement dans les normes juridiques de droit international. L'article $1^{\circ}$ de la Convention sur les Droits et Devoirs des États, signée lors de la Septième Conférence Internationale Américaine, célébrée à Montevideo en 1933, définit que : «L'État en tant que personne de Droit International doit remplir les exigences suivantes : I - Population permanente ; II - Territoire déterminé ; III - Gouvernement ; IV - Capacité d'entrer en relations avec les autres États ». La pratique internationale ajoute un élément de plus : la finalité établie dans sa Constitution. Il s'agit de l'élément social. Pour qu'un État existe et qu'il soit une personne de droit international, il doit remplir ces présupposés (support de fait) imposés par les normes de droit international.
} 
similitudes entre le droit interne et le droit international, et est répondue par la « norme de base » - règle de reconnaissance - qui valide les autres règles du système et en fait un système unique (HART, 1961, p. 287).

La question de l'absence de centralité dans l'application de la sanction est dépassée par Kelsen sans affecter la position de primauté du droit international. La sanction se révèle concept central dans la théorie kelsenienne du droit. C'est la clause qui sanctionne qui attribue aux normes le caractère de juridicité. La centralisation d'État différencie l'ordre interne des ordres supranationaux et internationaux. L'ordre international est essentiellement décentralisé.

S’il est possible de décrire le matériel qui se présente comme Droit international de telle façon que l'usage de la force par un État contre un autre ne puisse être interprété que comme délit ou sanction, alors le Droit international est Droit dans le même sens que le Droit national (KELSEN, 2005, p. 468).

Bien qu'il n'existe pas d'autorité unique, il y a toutefois le monopole du droit international pour appliquer les sanctions. C'est le droit international qui dira si un acte de guerre nationale sera considéré comme délit ou sanction internationale. La sanction du droit international est mise en rapport avec le degré d'interférence nationale dans les intérêts internationalement protégés d'autres États. Le délit, la représaille et la guerre sont des niveaux d'interférence que le droit international réglemente ; c'est pourquoi « la décentralisation de l'application du Droit n'empêche pas que l'acte coercitif en tant que tel soit strictement monopolisé » (KELSEN, 2005, p. 482).

Dans la position moniste internationaliste, il y a le Français Léon Duguit, qui, dans son analyse du Traité de Versailles (1919), renforce l'idée selon laquelle il reviendrait à l'OIT de développer une législation uniforme sur le travail. De cette façon, la législation internationale posséderait une force impositive et première sur les dispositions françaises concernant la législation du travail (DUGUIT, s/d, p. 160161).

Pontes de Miranda (1970, p.45) affirme que le Droit des Gens est un ordre normatif supérieur à l'État, d'où il retire sa personnalité et existence normative. Hors de l'ordre international, l'État ne demeure que dans sa dimension sociologique. La reconnaissance de l'existence de l'État se produit dans l'ordre supranational, selon les normes de droit international. Quant à l'ordre d'État, l' « être » État est organisé moyennant les normes constitutionnelles.

La primauté du droit international sur le droit interne découle 
de la force obligatoire des normes créées par les sujets de DIP dans un espace plus large que les territoires d'État - la société internationale. La tradition n'établit pas de hiérarchie entre les normes juridiques internationales, étant donné l'absence d'une autorité centrale semblable à la figure de l'État dans l'ordre interne. Toutefois, si hiérarchie signifie action de soustraire fondement et validité d'une norme d'une autre norme supérieure (et elle signifie cela même), il y a des normes juridiques internationales dont l'essence est si importante pour la conservation de l'humanité que sa force normative dépasse la formalité d'adhésion par les sujets de DIP. Ce sont des normes impératives.

Il s'agit d'une construction audacieuse au sein d'un système classiquement pensé et sédimenté dans la volonté des États, considérés comme étant les « réels » sujets de DIP. Le but de l'ordre international est de créer un cadre de protection de la personne humaine et de répandre ces règles sur les ordonnances nationales. Il est indéfendable de lui attribuer un objectif plus grand, bien que les actes internationaux continuent encore à être réglés selon les intérêts de l'État. Ici, le droit international (sens objectif) devient anachronique aux faits historiques qui ont prouvé l'insuffisance de l'action des Etats dans la protection de la vie humaine. C'est pourquoi quelques normes internationales méritent une observation impérative des sujets de DIP, spécialement les États, et doivent se positionner comme fondement des autres normes internationales, ainsi que des normes juridiques internes. L'engagement ne provient plus d'accords envers d'autres sujets de droit international, mais d'engagement envers la protection de toute vie humaine.

La prétention à étendre cette caractéristique à toutes les normes juridiques internationales serait utopique. Et ce parce que pas toutes les normes juridiques internationales ne se rapportent à l'essentialité de la vie humaine. Elle ne se tourne que vers les normes dont les effets attribuent à la personne humaine des pouvoirs susceptibles d'exiger la réalisation de prestations de qui a (de par la loi) le devoir et les facultés d'agir pour satisfaire ses intérêts, car cela lui sert au défi de développer ses potentialités en une existence digne. Cet ensemble normatif constituent les droits humains. Ainsi, les normes impératives se trouvent-elles inscrites dans une catégorie plus grande de normes juridiques internationales qui émanent des droits subjectifs dans sa charge d'efficacité (droits humains). La prévalence des droits humains est la norme de cette grandeur, mais il faut, avant d'approfondir davantage cette affirmation, considérer certains points relatifs aux droits humains.

\subsection{Traits remarquables de la primauté des droits humains}


Parmi les normes de jus cogens, se trouve la prévalence des droits humains. La primauté des droits humains donne impulsion à la reconnaissance des droits humains intangibles et, conséquemment, à la construction du noyau dur des droits humains. La prévalence sert de fil directeur à l'identification des normes impératives de DIP, tout comme elle empêche l'involution des droits humains déjà déclarés. Un autre point important, qui sera développé dans le prochain item, est la centralité de la personne humaine dans l'ordre international devant la personnalité juridique internationale de l'être humain. Même devant le renforcement des Organisations Internationales et de certaines collectivités non nationales, il y a longtemps que la société internationale est réglée selon la volonté et l'action des États comme « réels » sujets de DIP. Tout cela est caractéristique sous la perspective internationale.

Sous la perspective positiviste interne, la prévalence des droits humains se retrouve parmi les commandements apportés par l'article 4 de la Constitution Fédérale de 1988. Les Constitutions brésiliennes antérieures ne présentaient pas de dispositif semblable à celui-là, bien qu'il soit possible d'observer des préceptes rémanents dans toutes ces Constitutions, comme par exemple le principe de l'indépendance nationale ${ }^{2}$. Celle de 1988 innove, au moins en ce qui concerne la rédaction constitutionnelle choisie et, principalement, le contenu préliminaire, entendu ici comme une certaine notion de substance concédant une direction normative. Il suffit de lire le caput de l'article 4 pour comprendre (immédiatement) que le principe de la prévalence des droits humains possède comme destin premier la réglementation de la République Fédérative du Brésil dans ses relations internationales.

Les relations internationales dont traite l'article 4 ne doivent pas être confondues avec la science des Relations Internationales. L'article se penche sur la régulation des conduites de l'État par le biais d'un système normatif-coactif (objet des sciences juridiques), alors que les RI voient les rapports de pouvoir parmi divers acteurs sociaux et politiques à partir de la faveur et de l'intérêt comme " monnaie d'échange " (objet des sciences politiques) (CASTRO, 2012, p. 280). De plus, sous l'aspect juridique, l'État est sujet, tandis que pour les RI il est acteur, c'est-à-dire, possédant entière capacité d'influence directe ou indirecte dans la politique internationale à la lumière des $\mathrm{Kfpi}^{3}$ (CASTRO, 2012, p. 429). Les sujets internationaux sont « liés à l'ethos dans le champ de la légalité et de la légitimité ». Soumis et sujets à l'ensemble de normes juridiques en vigueur dans un système international déterminé

2 Il ne serait pas cohérent de penser que le concept d'indépendance nationale présent dans la Constitution Impériale de 1824 est le même que celui qui se trouve dans la Constitution Fédérale de 1988. Les contenus sont rénovés par la nouveauté constitutionnelle face aux changements sociaux.

3 Capitales de force-pouvoir-intérêt. 
(CASTRO, 2012, p. 430).

Outre le principe de la prévalence des droits humains, la Constitution Fédérale apporte explicitement dix principes : a) indépendance nationale ; b) auto-détermination des peuples ; c) nonintervention ; d) égalité entre les États ; e) défense de la paix ; f) solution pacifique des conflits ; g) répudiation du terrorisme et du racisme ; h) coopération entre les peuples pour le progrès de l'humanité ; i) concession d'asile politique. En tout, donc, dix principes explicites et, parmi les implicites, il y en a un assez évident dans le paragraphe unique concernant la recherche de l'intégration économique, politique, sociale et culturelle des peuples de l'Amérique Latine, dans le but de former une communauté latino-américaine d'États.

Du modèle classique de Constitution jusqu'au modèle le plus garantiste, l'organisation de l'État et la réglementation des droits et des libertés de la personne humaine ont toujours été matière constitutionnelle. Dans le constitutionnalisme contemporain, ces droits remplissent deux fonctions : subjective et objective. La fonction subjective implique la protection des libertés individuelles. La fonction objective assume une dimension institutionnelle. À travers celle-ci, ses contenus doivent fonctionner comme directives pour les finalités constitutionnellement proclamées (PÉREZ LUÑO, 2004, p. 19-22).

Les chartes constitutionnelles plus analytiques ne se contentent pas de réglementer la division de l'État, dans le sens de n'établir que ses compétences. Elles vont au-delà. En positivant, par exemple, les principes de coexistence pacifique entre les sujets de la société internationale, les Constitutions normativent la politique étrangère de leurs pays. Les représentants d'un État décident comment celui-ci devra s'insérer dans la société internationale, conjugant idéaux et intérêts. Au Brésil, par exemple, lors de sa prise de fonction présidentielle du premier mandat, le Président Lula affirmait que l'action diplomatique de son gouvernement serait orientée par « une perspective humaniste », servant d' « instrument de développement national » et, tournée vers ce développement et la paix, la politique étrangère chercherait à « réduire le hiatus entre nations riches et nations pauvres, promouvant le respect de l'égalité entre les peuples et la démocratisation effective du système international »- en lignes générales (BARRETO, 2012, p. 7).

Au premier coup d'oeil, on peut penser que les actes d'État délimités par la recherche de ressources de pouvoir relèvent du libre choix de ceux qui le représentent. Il n'en est rien. La souveraineté populaire qui donne naissance au pacte constituant et au principe démocratique qui en découle, et les fonde, a une influence importante dans le processus de constitutionnalisation des relations internationales. La démocratie est l'une des principales causes de la réglementation normative et constitutionnelle des relations étrangères (DALLARI, 1994, 
p. 13). Elle fonde la nécessité d'écarter le caractère obscur qui marquait historiquement les relations d'État. Le pouvoir qui émane du peuple lui assure le droit de savoir et de participer aux choix de son État, et même de les contrôler. Ainsi, l'ordonnance juridique brésilienne a-t-elle constitutionnalisé les relations internationales en dépit de la résistance historique que manifeste la politique étrangère à la participation et au contrôle démocratique. L'élargissement du traitement constitutionnel des relations étrangères est une caractéristique des Constitutions modernes.

L'abordage purement administrativiste de cette réglementation affirme que la Constitution Fédérale remplit la fonction d'établir des règles de procédés et de paradigmes devant être en vigueur « [...] parallèlement au déploiement d'initiatives subordonnées à la politique étrangère $d u$ gouvernement », assurant la viabilité de surveillance de la part de la société (DALLARI, 1994, p. 16). Elle se matérialise devant l'inexistence ou le manque d'importance de l'orientation effective légale (BROTÓNS, 1984, p. 13). Antonio Ramiro Brotóns identifie dans la fixation de jalons normatifs de la gestion politique étrangère, dans l'établissement de limites à la politique étrangère et dans la formulation de stimuli tournés vers l'orientation de la politique étrangère, afin d'atteindre les buts proposés, les trois fonctions de cette constitutionnalisation (1984, p. 93-103).

Ce qui intéresse ici c'est la fonction normative de l'article 4 de la Constitution Fédérale - de manière générale - ciblant la prévalence des droits humains qui ne se lie pas seulement à l'organisation de la gestion politique étrangère. La prévalence des droits humains qui régit la République Fédérative du Brésil dans ses relations internationales doit être travaillée au-delà de sa perspective purement administrativiste.

Les principes des relations internationales, tels qu'ils se trouvent dans le texte constitutionnel, manifestent le processus de consolidation et d'évolution de la démocratie au Brésil. Toutefois, ils révèlent indéniablement une référence à la Déclaration de 1970. Il s'agit de la Déclaration Relative aux Principes du Droit International Régissant les Relations Amicales et la Coopération entre États Conformément à la Charte de 1'ONU. Cette Déclaration, dans sa partie générale, souligne que ses principes sont liés entre eux et constituent des normes de base de droit international. Elle renforce aussi l'importance des États à internaliser ces précepts (TRINDADE, 1981, p. 52-80).

Dans les antécédents historiques de la Déclaration de 1970 se trouvait le phénomène de la décolonisation. L'indépendance conquise par divers peuples, créant de nouveaux États, a modifié profondément les relations internationales. Ce phénomène a donné de l'impulsion à la normatisation de ces principes. L'évolution du concept de coexistence pacifique de tous les États a également contribué à la Déclaration en 
question. Il convient encore de souligner qu'elle n'est pas apparue comme un amendement à la Charte de l'ONU, mais comme une interprétation de ses principes à la lumière des finalités proposées par les Nations Unies (TRINDADE, 1981, p. 52-80).

La Déclaration de Principes de Droit International relative aux Relations Amicales et à la Coopération entre États conformément à la Charte de l'ONU a été adoptée le 24 octobre 1970, dans la Résolution 26/25 (XXV), par l'Assemblée Générale Plénière présidée par M. Edvard Hambro (Norvège), à l'occasion de la séance commémorative du vingt-cinquième anniversaire des Nations Unies. Dans un passage du discours de M. Hambro, on peut subtilement percevoir une mention aux idées contraires à la Déclaration de $1970^{4}$.

\begin{abstract}
[...] As a man of law I am particularly happy to have just announced the adoption of the Declaration on Principles of International Law concerning Friendly Relation and Co-operation among States in accordance with the Charter of the United Nations. This marks the culmination of many years of effort for the progressive development and codification of the concepts from which basic principles of the Charter are derived. The Assembly will remember that when we first embarked upon these efforts many doubted that it would be acceptable to all the various political, economic and social systems represented in the United Nations. Today those doubts have been overcome. In a sense, however, the work has just begun. We have proclaimed the principles; from now on we must strive to make them a living reality i the life of States, because these principles lie at the very heart of peace, justice and progress $^{5}$.
\end{abstract}

4 Disponible sur : http://untreaty.un.org/cod/av1/ha/dpilfrcscun/dpilfrcscun.html. Accès le 20/07/2014.

5 En qualité d'homme de droit, je suis particulièrement heureux car je viens d'annoncer l'adoption de la Déclaration des Principes de Droit International relatifs aux Relations d'Amitié et de Coopération entre les États, conformément à la Charte des Nations Unies. Ceci marque le point culminant de nombreuses années d'effort pour le développement base de cette Charte. L'Assemblée se rappelle sans doute que, quand nous avons entrepris ces efforts, beaucoup ont douté qu'il serait possible d'obtenir un résultat acceptable aux divers systèmes politiques, économiques et sociaux représentés aux Nations Unies. Aujourd'hui, ces doutes ont été surmontés. En un certain sens, cependant, le travail ne fait que commencer. Nous avons proclamé les principes ; dès maintenant, nous devons redoubler d'efforts pour les transformer en réalité vivante dans les États, car ces principes sont au coeur de la paix, de la justice et du 
Avec davantage d'emphase, le président norvégien de l'assemblée Générale de l'ONU a affirmé l'importance de mener ces principes déclarés à la réalité (de fait et juridique) de tous les États. Les débats dans cette sphère - nommée effort dans le champ de la codification et du progressif développement du droit international - ont migré de la Sixième Commission de l'ONU à l'agenda provisoire de l'Assemblée Générale lors de la $17^{\mathrm{e}}$ séance, par la Résolution 1.686 - XVI, le 18 décembre 1961, intitulée " Consideration of principles of international law concerning friendly relations and co-operation among States in accordance with the Charter of the United Nations ». Sitôt reconnue l'importance des sept principes de droit international sur les relations amicales et la coopération entre États (Résolution 1.815 - XVII, du 18 décembre 1962), des études ont été entamées pour les développer et les codifier. C'est pour cette raison que l'Assemblée Générale a créé la Commission Spéciale de 1964 (Résolution 1.966 XVIII, du 16 décembre 1963).

À partir de 1966 jusqu'en 1969, la Commission Spéciale s'est réunie annuellement ${ }^{6}$. Les rapports présentés par la Commission révélaient une discordance quant à la signification des principes, spécialement en quoi consisterait le recours à la force ${ }^{7}$. A la fin des débats, l'Assemblée Général a adopté la Déclaration retenant les principes suivants : a) principe selon lequel les États doivent éviter dans

progrès. (Traduction de l'auteure).

New York, du 8 mars au 25 avril 1966 ; Genève, du 17 juillet au 19 août 1967 ; New York, du 9 au 30 septembre 1968 ; New York, du 18 août au 19 septembre 1969 (présentant les rapports respectifs à l'Assemblée Générale - A/6.230, A/6.799, A/7.326 et A/7.619).

Les rapports de la Commission Spéciale de 1964 n’ont pas été trouvés sur le site officiel de l'ONU. Ce n'est que par le biais de Netherlands School of Human Rights Research qu'il a été possible d'accéder aux textes originaux. Disponible sur : http://invisiblecollege.weblog. leidenuniv.nl/2010/03/03/special-committee-on-principles-of-inter. Accès le 26/07/2012. progressif et la codification des concepts d'où dérive chaque principe de base de cette Charte. L'Assemblée se rappelle sans doute que, quand nous avons entrepris ces efforts, beaucoup ont douté qu'il serait possible d'obtenir un résultat acceptable aux divers systèmes politiques, économiques et sociaux représentés aux Nations Unies. Aujourd'hui, ces doutes ont été surmontés. En un certain sens, cependant, le travail ne fait que commencer. Nous avons proclamé les principes ; dès maintenant, nous devons redoubler d'efforts pour les transformer en réalité vivante dans les États, car ces principes sont au coeur de la paix, de la justice et du progrès. (Traduction de l'auteure).

6 New York, du 8 mars au 25 avril 1966 ; Genève, du 17 juillet au 19 août 1967 ; New York, du 9 au 30 septembre 1968 ; New York, du 18 août au 19 septembre 1969 (présentant les rapports respectifs à l'Assemblée Générale - A/6.230, A/6.799, A/7.326 et A/7.619).

7 Les rapports de la Commission Spéciale de 1964 n'ont pas été trouvés sur le site officiel de l'ONU. Ce n'est que par le biais de Netherlands School of Human Rights Research qu'il a été possible d'accéder aux textes originaux. Disponible sur : http://invisiblecollege.weblog. leidenuniv.n1/2010/03/03/special-committee-on-principles-of-inter. Accès le 26/07/2014. 
leurs relations internationales le recours à la force contre l'intégrité territoriale ou l'indépendance politique de n'importe quel État ou de n'importe quelle manière incompatible avec les intentions des Nations Unies ; b) principe selon lequel les États doivent résoudre leurs disputes internationales par des moyens pacifiques, de telle forme que la paix, la sécurité et la justice ne se trouvent pas en péril ; c) devoir de nonintervention dans des affaires de juridiction domestique de n'importe quel État, selon la Charte des Nations Unies ; d) devoir de coopération mutuelle entre États ; e) principe d'égalité de droits et d'autodétermination des peuples ; f) principe d'égalité de souveraineté des États; et g) principe selon lequel les États doivent remplir de bonne foi leurs obligations assumées conformément à la Charte des Nations Unies, et aussi assurer leur plus grande effectivité dans la société internationale afin de réaliser les intentions des Nations Unies.

La prévalence des droits humains est un principe constitutionnel, internalisé après intense mouvement international en faveur de la déclaration de principes de base susceptibles de guider les États dans leurs relations internationales. La compréhension du principe de prévalence des droits humains passe par deux niveaux importants. Ces degrés de perception normative se révèlent dans deux questions principales. D'abord, qu'est-ce qui doit prévaloir ? La réponse à cette interrogation se trouve facilement dans la structure du principe luimême. Elle est évidente dans les lectures les moins prétentieuses prévalence des droits humains. Ici, la commande normative est entière, perçue comme un tout, synthétiquement analysé. Ce sont les droits humains qui devront prévaloir. Telle est l'exigence normative.

Les droits humains forment un univers complexe d'idées et, en même temps, ils composent, dans une vision plus holistique ${ }^{8}$, la

8 Le mot holon est d'origine grecque (ő $\lambda \mathrm{ov}$ ) e a été employé pour la première fois par le philosophe Arthur Koestler dans The Ghost in the Machine. Le concept a été bien expliqué par Yves Bouchard dans la note 2 de bas de page du livre Le Holisme épistémologique de Kant : Parmi les représentants de la thèse réductionniste, on compte entre autres Russel et le Wittgenstein duTractatus, et parmi les représentants de la thèse holiste, Quine (holisme épistémologique) et Davidson (holisme sémantique) en particulier. Cette opposition est également fréquente dans d'autres disciplines. D'un point de vue sociologique, une société d'individus ne se réduit pas au seul nombre d'individus qui la composent. La société possède une dynamique propre dont on ne peut rendre compte par la seule énumération des individus qui en font partie. Par ailleurs, ce n'est pas non plus en regroupant spontanément un certain nombre d'individus que par là on forme une société. En génétique, l'étude des systèmes adaptatifs complexes repose en bonne partie sur cette conception d'un système comme un tout, c'est-à-dire que le système lui-même possède des propriétés exclusives que l'on ne saurait rencontrer ni dans les éléments, ni dans leur simple conjonction. A. Koestler a donné le nom de Holon ( du grec ó̉ov) à cette forme particulière d'unité. (BOUCHARD, [S.d.], p. 13). 
direction de l'ordre de prévalence de la primauté des droits humains. Ainsi, enquêter sur le concept de primauté des droits humains c'est, en même temps, se positionner sur les droits humains qui vont prévaloir. Il convient de souligner que la signification et l'éclaircissement des droits humains renforcent l'idée de la nécessité d'une dialectique plus démocratique, ou, en d'autres termes, selon Karl Raimund Popper (1998, p. 19), renforcent la construction d'une méthode scientifique ayant des prémisses qui n'aient pas l'intention d'être des vérités absolues, substitut de la certitude scientifique par le progrés scientifique.

Selon les concepts abordés dans cette recherche, la réponse à la question posée antérieurement se révèle graduelle au fur et à mesure que certaines garanties retombent sur les droits humains. Il y a des droits humains reconnus dans le droit international, des droits humains reconnus et intangibles, des droits humains reconnus, intangibles et communs du noyau dur et, au degré de primauté le plus élevé, des droits humains de ius cogens. Dans ce sens, la primauté rend l'instrumentalité des droits humains. Soutenir des normes protectrices de la personne humaine qui seront toujours au-dessus des entraves formalistes et des gouvernements qui ne respectent pas ou qui ne ratifient même pas les traités internationaux sur les droits humains, cela signifie stimuler le processus de renforcement du droit international des droits humains. Il s'agit d'échanger la recherche des vérités scientifiques contre une dialectique plus ouvertes aux critiques. Il est indispensable de souligner que le cadre idéal à cette activité d'étoffement constant du ius cogens est la démocratie. Dans celle-ci, les préceptes seraient analysés et construits par tous les sujets - collaborateurs des valeurs démocratiques -, ciblant la nécessité de rendre compatibles les contenus avec la protection de la personne humaine.

Il incombe à la prévalence des droits humains, en tant que norme de ius cogens, d'impulser la construction du noyau dur des droits et, conséquemment, l'idée des droits humains communs. La reconnaissance de la personne juridique internationale de la personne humaine, centralisée par la force première des droits humains, doit se sédimenter dans l'ordre international afin de démontrer le retard des positionnements qui empêchent encore la participation entière de l'être humain dans la société internationale. C'est de la personnalité de DIP de la personne humaine que découle la nécessité de réformer les règles processives internationales qui, dans la pratique, se révèlent contradictoires dans leur participation en qualité de sujet.

\section{LA FORCE IMPERATIVE DE LAPREVALENCE DES DROITS NUMAINS ET LE RETOUR DE LA PERSONNE HUMAINE AU CENTRE DU DROIT INTERNATIONAL : D'OBJET A SUJET DE DIP}


La vision marquée par la souveraineté absolue des États demeure encore forte dans certains champs du droit international (spécialement dans la création de normes juridiques internationales, telle que la formation d'accords et de postulats dans les tribunaux internationaux). La souveraineté absolue s'unit à l'image d'un État absolu, hautain et étranger à l'existence de sujets n'assumant pas la forme d'un État. Cette image est incompatible avec la position remarquable des Organisations internationales, ainsi qu'avec l'action importante de la personne humaine dans les espaces internationaux.

La personnalité de DIP des Organisations internationales a entamé sa sédimentation à partir des positionnements du Tribunal International de Justice concernant les réparations pour dommages dans l'affaire de l'assassinat du comte Folke Bernadotte, premier médiateur des Nations Unies en palestine, le 17 septembre 1948. On s'est demandé si l'ONU était détentrice de personnalité pour demander réparations pour l'assassinat du diplomate suédois. La position de M. Felleb s'exprime dans le discours incisif de la cour concernant la personnalité des Nations Unies :

\section{[...] comme il a été démontré, la personnalité internationale des Nations Unies est fermement établie dans le droit international, non seulement dans les préceptes de la Charte comme un tout, mais aussi dans la pratique des États, membres et non membres. Je dois à présent démontrer que cette personnalité porte en elle-même la capacité nécessaire à l'accomplissement de ses intentions et l'exercice de ses fonctions du point de vue procédural .}

Un autre sujet dont la personnalité se renforce dans la phase $\mathrm{du}$ droit international post-Seconde Guerre Mondiale est la personne humaine. José Roberto Franco da Fonseca analyse la géopolitique et le droit international et soutient que les États ont de plus en plus besoin d'établir des relations avec les gens, sous une perspective physique ou juridique. Franco da Fonseca identifie le rapport de l'État avec les étrangers qui entrent dans son territoire, avec les étrangers

$9[\ldots]$ as we have just shown, the international personality of the United Nations is firmly established in international law, not only by the Charter provisions as a whole, but also by State practice on the part of those Member and non-member States. I should now like to demonstrate that this personality carries with it the capacity necessary for the fulfilment of its purposes and the exercice of its functions from a procedural standpoint. Statiment by $\mathrm{Mr}$. Felleb (United Nations) p. 74-7, III, 1949. Disponible sur : http://www.icj-cij.org/search/index. php?pg=1\&p2=2\&op=0\&str=+Conde+Folke+\&1g=0\&op=0. Accès le 22/01/2014. 
représentants d'États, avec des nationaux qui se rendent dans d'autres États, ainsi que la relation de l'État avec ses habitants et la relation de la société internationale d'États avec les personnes, devant l'imputation de devoirs envers elles (crimes internationaux ou crimes de caractère international) (1996, p. 319-320). D'objet à sujet, la personne humaine cesse de participer indirectement à la société internationale et devient destinataire de droits et de devoirs de DIP.

En 1949, Câmara Filho n'écartait pas la possibilité de reconnaissance de la personnalité internationale de la personne humaine. L'auteur argumentait que les personnes ne se verraient « en situation d'apparaître comme sujets de droit international, directement obligés ou autorisés par la norme, que lorsqu'existeraient des cours internationales devant lesquelles ils pourraient paraître comme plaignants (1949, p. 102). Sous cette perspective, il serait déjà possible de voir la personne humaine comme sujet de DIP dès 1907, quand a été créée la Cour de Justice Centre-Américaine. La CJCA, dont le fonctionnement a commencé en 1908, a été créée dans un contexte d'instabilité des relations des pays d'Amérique Centrale, en raison de la forte intervention de puissances extra-régionales. La célèbre Doctrine Monroe, dans la pratique, a représenté les efforts déployés par les États-Unis pour subjuguer les États d'Amérique Centrale en faveur des intérêts américains, l'un d'eux étant la construction d'un canal interocéanique dans la région.

Au cours des Conférences de Washington, les États d'Amérique Centrale ont signé le Traité Général de Paix et Amitié, la Convention Additionnelle au Traité Général, la Convention qui créaient la Cour de Justice Centre-Américaine et autres six accords. Selon SORTO, la CJCA a été le premier organisme doté d'une juridiction aussi large que la juridiction des tribunaux domestiques, contrairement à la Cour Internationale de Justice de La Haye (CIJ), dont la compétence est limitée par la volonté des États (SORTO, 1999, p. 293). La CJCA constituait une grande avancée dans la perception de la personne humaine en tant que sujet de droit international parce qu'elle permettait, selon sa compétence, des requêtes de citoyens d'Amérique Centrale contre les gouvernements des États-parties, face à l'épuisement des instances internes ou aux dénégations de justice par le gouvernement requis. Il incombait également à cette Cour de juger les requêtes non résolues par les chancelleries des États intéressés. Bien que l'existence de la Cour de Justice Centre-Américaine ait été plutôt brève, la requête en jugement du nicaraguayen Alejandro Bermúdez Núñez contre le Costa-Rica est allée jusqu'au bout. Sans aucun doute, la CJCA a été pionnière en donnant un accès juridictionnel à la personne humaine.

La centralité de la personne humaine dans l'ordre international, consubstancielle à l'attribution de personnalité juridique de DIP, 
a été analysée par SORTO dans sa recherche sur la condition de la personne humaine dans le Projet de Code de Droit International Public d'Epitácio Pessoa, élaboré en 1911. Pour Sorto, l'auteur du projet s'est aligné sur la perception classique de la position des États en tant qu'uniques sujets de droit international, laissant de côté des avancées déjà perçues à l'époque. En 1911, année de l'élaboration du projet, la Cour de Justice Centre-Américaine était déjà opérante (1907-1918) et sa jurisprudence reconnaissait la personne humaine en tant que sujet de droit international, lui permettant de figurer comme partie dans les actions. Actions qu'elle pourrait traduire en justice contre l'État de sa nationalité (SORTO, 2013, p. 145-146).

Une autre avancée est constituée par les contributions de doctrinaires tel que Pasquale Fiore, lequel considérait sujet de droit international tout être ou institution ayant une individualité créée de son propre droit et agissant dans le monde (FIORE apud SORTO, 2013, p. 141-142). D'autres auteurs s'alignent sur Fiore et Sorto, tels Alejandro Álvarez, Hildebrando Accioly, Haroldo Valladão et Cançado Trindade. L'État ne doit pas être compris comme étant une fin en soi-même, mais comme une structure créée par des personnes, dont l'existence n'est légitimée que dans la recherche de leur protection. La personne humaine ne peut donc être soumise à la volonté de l'État, pas plus que le droit international ne peut être fondé uniquement sur la volonté des États. Pour Francisco de Vitoria, l'un des fondateurs du droit international, dans son ouvrage De Indis (chapître VI et VII), le droit international régule une société internationale composée d'êtres humains organisés socialement en États. De plus, les réparations des violations aux droits humains caractérisent une nécessité internationale atteinte par le droit international avec les mêmes principes de justice appliqués aussi bien aux États qu'aux personnes. Le droit des gens est un droit pour tous, personnes et États (VITORIA apud TRINDADE, 2014, p. 253).

L'action de la personne humaine est indispensable à la concrétisation des droits humains parce qu'elle configure l'essence du concept commun de droits humains, développé au cours de cette recherche. La création de tribunaux internationaux marque bien cette transformation. Outre l'expérience de la Cour de Justice CentreAméricaine pour ce qui est de la participation de la personne humaine, il est possible actuellement de mentionner la Cour Européenne de Droits Humains, la Cour Interaméricaine de Droits Humains et le Tribunal Pénal International. Chacun représente, avec sa systématique propre, un espace d'action de la personne humaine dans l'ordre international, que ce soit en qualité de requérant (Cour Européenne de Droits Humains et - indirectement - la Cour Interaméricaine de Droits Humains) ou en qualité inverse (Tribunal Pénal International, dans les cas de crimes contre l'humanité, crimes de guerres et génocide). Il convient d'ajouter 
encore la pratique récente du Conseil de Sécurité, reconnue par la Cour Internationale de Justice, de créer des obligations à des entités n'étant pas d'État, y compris des individus (PARLETT, 2010, p. 298), ainsi que le Protocole Facultatif du Pacte de Droits Civils et Politiques qui rend viable, moyennant le consentement de l'État soumis à requête, l'accès de la personne humaine victime de violations de droits humains à la Cour Internationale de Justice.

La personnalité juridique nationale et celle qui est internationale sont des attributs de l'être humain, car le droit international se base sur la prévalence des droits humains et, conséquemment, s'appuie sur la centralité de la personne humaine. Les droits humains communs, la primauté des droits humains et toutes les autres normes de ius cogens outrepassent la figure des États et se lancent directement dans l'action et dans la protection des personnes. Si des arguments contre la personnalité internationale de la personne humaine trouvent encore quelque appui c'est parce qu'il existe encore un attachement rétrograde à la souveraineté absolue des États. La pratique ne chemine pas en synchronie avec la protection de la personne humaine des droits humains. Cela exige une recherche du niveau de participation de la personne humaine dans les tribunaux de droits humains en vigueur, sous la perspective critique de la prévalence des droits humains.

\section{CONCLUSION}

Les discours purement rhétorique par les États ne seront retranchés des relations internationales, comme le prouve lorsque la participation de l'individu dans ces relations est le fait efficace. En fin de compte, il y a encore de nombreux obstacles à des préjugés politiques et juridiques. Par conséquent, le principe de la prévalence des droits de l'homme est apparue comme une véritable ligne directrice du comportement de l'État, que ce soit au niveau international, que ce soit en interne. En raison du principe de la prévalence des droits de l'homme sont nés en droit international, ce qui lui donne le statut de primate norme sur le droit interne, comme le montre.

La primauté du droit international sur le droit national résulte de la force obligatoire des normes créées par les sujets DIP dans la société internationale. Cependant, la nature juridique de ce principe était au-delà des règles « typiques » du droit international, on a vu que sa pertinence normatif axé sur les besoins historiques et sociaux pour protéger les droits de l'homme soulevées à la catégorie des jus cogens. Il a été démontré que la force probante signifie l'imposition au-delà de la volonté des Etats comme le manque d'intérêt des États à l'égard des droits de l'homme reste visible à travers les nombreuses condamnations dans les tribunaux internationaux des droits, sans parler des plaintes 
aux organes compétents.

Ce manque d'intérêt a également été noté dans le domaine des normes de jus cogens parce que même si elles ont été créées en tant que catégories, il n'y avait pas d'avance législative au contenu de l'accomplissement. Soit dit en passant, le thème est encore très délicate, même quand il a parlé de remplissage jurisprudentielle des tribunaux internationaux. Selon ce qui a été dit, le ius cogens existe pour répondre aux intérêts supérieurs de la société internationale, et non pas les intérêts des Etats, alors l'existence valide des obligations erga omnes DIP. Le fait que les législateurs et les juges internationaux demeurent quant à savoir si cette catégorie normes si particulières, légitimant davantage le rôle de la doctrine de pointer les normes qui composent le jus cogens. Cela a été fait ici.

La nature convaincante de la primauté des droits de l'homme a conduit au domaine controversé sur l'étendue des droits de l'homme, parce que si le point tente de mettre en cause la légitimité même des droits de l'homme. On a remarqué que le relativisme plutôt affaibli l'idée de l'universalité des droits de l'homme, parce que dans les États ou les groupes sociaux de refuser de mettre en œuvre des droits de l'homme au motif que ces dispositions font mal ses traditions, la culture, etc. Cependant, on a constaté qu'il n'y a pas d'arguments découlant des aspects relativistes justifiant l'évincement de certains droits individuels.

Alors que certains ont fait des droits sacrés que les barrières universelles à sa trouvaille longueur, on a vu que les droits subjectifs capables de former un noyau de droits de l'homme commun à tous. La commune correspond donc à l'espace dans lequel tous sont ou souhaitent faire partie, l'espace est divisé entre eux et où tout le monde participe. Voici le lien à l'action politique. Ainsi, la commune est déduite ne peut légitimer progressivement à partir des noyaux plus simples (famille, locale) au plus complexe (international), déférent universel qui a l'extension comme son teint.

Par conséquent, il est conclu que le noyau des droits de l'homme a été construit par les principaux traités de droit international qui forment les soi-disant systèmes internationaux de protection de la personne humaine. Il est très élevé, car l'obtention du diplôme traditionnellement considérés comme domestiques dépassé les frontières de l'État et des sujets atteints lorsque relatif à la protection des êtres humains. Il est devenu clair que la création de ce besoin impérieux d'une plus grande participation de l'individu dans l'ordre international, il est « né à la politique étrangère. " Tous les arguments et les règles qui ont été présentés comme des obstacles à ces actions des êtres humains étaient simplement des outils de l'Etat.

Il ne serait pas raisonnable de croire que les actes de l'État toujours signifierait la réalisation du contenu essentiel de la personne 
humaine. Ainsi, selon le durable, le principe de la primauté revient l'instrumentalité des droits de l'homme, a défendu l'existence de normes de protection de la personne humaine qui sont toujours audessus des barrières formalistes et les gouvernements, comme on le voit, violent ou non à défaut de ratifier et d'adhérer aux traités relatifs aux droits de l'homme.

L'assimilation négative de certains groupes de droits de l'homme universels n'a pas le même sens de l'assimilation négative des droits de l'homme ordinaire. Dans le premier cas, il la question présentée par le rapport correspondant à l'abstraction simple. L'analyse du second cas a exigé une plus grande prudence. assimilation des non-droits de l'homme commun signifiait la négation de la possibilité de la vie humaine socialement partagée. Le déni de l'humanité ellemême est la force essentiel dans les crimes de masse. Le discours des conséquences de ne pas l'assimilation aux droits de l'homme commun pourrait être perçu dans diverses situations de violence qui ont dans le texte. Le principe de la primauté des droits de l'homme est contraire aux privilèges, parce qu'ils nient essentiels à la construction et à la reconnaissance de cette égalité de règle. De sorte que, par l'action politique, sont réunis les droits communs à tous est nécessaire que ceux qui agissent sur le plan politique sont sur un pied d'égalité. On a vu qu'il est possible que de faire de la politique dans la zone commune, à savoir où il y a égalité. De même, que si elle est libre dans l'espace qui agit sur le plan politique.

\section{RÉFÉRENCES}

ARBUET-VIGNALI, Heber. Peligrosos prejuicios sobre soberanía. In: Anuario de derecho constitucional latinoamericano. Tomo I. Uruguay: Fundación Konrad-Adenauer, 2006, p. 65-104.

ARENDT, Hannah. La crise de la culture: huit exercices de pensée politique. Traduit de l'anglais sous la direction de Patrick Lévy. Trebaseleghe: Éditions Gallimard, 2012.

. Origens do totalitarismo: Antissemitismo, imperialismo, totalitarismo. Traduit par Roberto Raposo. São Paulo: Companhia das Letras, 2012.

. La condición humana. Traducido por Ramón Gil Novales. Buenos Aires: Paidós, 2012.

. A promessa da política. Traduit par Pedro Jorgensen Jr. Rio de Janeiro: 2008.

- ¿Qué es la política? Traducido por Rosa Sala Carbó. Barcelona: Paidós, 1997. 
ARIZA, Santiago Sastre.Lacienciajurídicaante elneoconstitucionalismo. In: CARBONELL, Miguel (ed.). Neoconstitucionalismo(s). 2. ed. Madrid: Trotta, 2005, p. 239-258.

ATIENZA RODRÍGUEZ, Manuel. Marx y los derechos humanos. Lima: Palestra, 2008.

BACHOF, Otto. Normas constitucionais inconstitucionais? Traduit par José Manuel M. Cardoso da Costa. Coimbra: Almedina, 1994.

BIELEFELDT, Heiner. Filosofia dos direitos humanos: fundamentos de um ethos de liberdade universal. Traduit par Dankwart Bernsmüller. São Leopoldo: Unisinos, 2000.

BOBBIO, Norberto. Teoria do ordenamento jurídico. Tradução de Maria Celeste Cordeiro Leite dos Santos. 6. ed. Brasília: Editora da Universidade de Brasília, 1995.

. Teoria da norma jurídica. Tradução de Fernando Pavan Baptista e Ariani Bueno Sudatti. 2. ed. São Paulo: EDIPRO, 2003.

BOSON, Gerson de Brito Mello. O homem como sujeito de direito internacional. Belo Horizonte: [S.n.], 1951.

BOUTMY, Émile. La déclaration des droits de l'homme et du citoyen et M. Jellinek. In: Études politique. Paris: Armand Colin, 1907, p. 119182.

BROWNLIE, Ian. Princípios de direito internacional público. Traduit par Maria Manuela Farroja. et al. Lisboa: Fundação Calouste Gulbenkian, 1997.

BURDEAU, Georges. Les libertes publiques. 2. ed. Paris: R. Pichon e Durand-Auzias, 1961.

CÂMARA FILHO, José Sette. Hans Kelsen e a teoria pura do direito internacional. Revista da Faculdade de Direito. Belo Horizonte: Universidade de Minas Gerais, p. 77-113 out 1949.

CASTRO, Thales. Teoria das relações internacionais. Brasília: FUNAG, 2012.

COLLOQUE DE LILLE. La juridictionnalisation du droit international. Paris : A. Pedone, 2003.

DALLARI, Dalmo de Abreu. Elementos de teoria geral do Estado. 3. ed. São Paulo: Saraiva, 1982.

DALLARI, Pedro. Constituição e relações exteriores. São Paulo: Saraiva, 1994.

DAUSSET, Jean. Le droit à la différence. In: Pourles droits de l'homme: Mélanges en l'honneur de l'ADLF. [S.1.]: Librairie des libertes, 1983, 
p. 55-72.

DUGUIT, Léon. Traité de Droit Constitutionnel. 2. ed. vol. 5. Paris: Éditions Cujas, [S.d.].

DURÃES, Beatriz Schiffer. O indivíduo: sujeito de direito internacional público? In: Revista da Faculdade de Direito da UFPR. Curitiba, a. 28, n. 28, 1994/1995, p. 141-148.

DWORKIN, Ronald. Una cuestión de principios. Traducción de Victoria de los Ángeles Boschiroli. Buenos Aires: SigloVeintiuno, 2012. ESMEIN, A. Élément de droit constitutionnel français et comparé. Paris: Panthéon-Assas, 2001.

ESPIELL, Héctor Gros. La prevención de conflictos bélicos en el Derecho Internacional actual. Las Naciones Unidas y el sistema interamericano. In: Anuario de derecho constitucional latinoamericano. Tomo I. Uruguay: Fundación Konrad-Adenauer, 2002, p. 375-392.

ESPIELL, Héctor Gros. El derecho humano a la paz. In: Anuario de derecho constitucional latinoamericano. Tomo I. Uruguay: Fundación Konrad-Adenauer, 2005, p. 517-546.

FERNÁNDEZ GARCÍA, Eusebio. El problema del fundamento de los derechos. Anuario de derechos humanos - 1981. Madrid: Instituto de Derechos Humanos de la Facultad de derecho, Universidad Complutense Madrid-3, 1981, p. 77 - 112.

. Dignidad humana y ciudadanía cosmopolita. Madrid: Instituto de derechos humanos Bartolomé de las Casa Universidad Carlos III de Madrid/Dykinson, 2001.

FONSECA, José Roberto Franco da. Geopolítica e direito internacional. Revista da Faculdade de Direito. São Paulo: Universidade de São Paulo, p. 315-329 v. 91, 1996.

GARCÍA-PELAYA, Manuel. Derecho constitucional comparado. Madrid: Alianza, 1999.

GUTMANN, Amy. La identidad en democracia. Buenos Aires: Kutz, 2008.

HABERMAS, Jürgen. Sobre a constituição da Europa: um ensaio. Traduit par Denilson Luis Werle, Luiz Repa e Rúrion Melo. São Paulo: Unesp, 2012.

HART, Herbert L. A. El concepto de derecho. Traducción de Genaro R. Carrió. Buenos Aires: Abeledo-Perrot, 1961.

HART, H.L.A. Utilitarismo y derechos naturales. Anuario de derechos humanos - 1981. Madrid: Instituto de Derechos Humanos de la Facultad 
de derecho, Universidad Complutense Madrid-3, 1981, p. 149-168.

HAURIOU, Maurice. Principios de derecho público y constitucional. 2.ed. Traducido por Carlos Ruiz del Castillo. Madrid: Centro de Enseñanza y Publicaciones, [S.d.].

HERRERA FLORES, Joaquín. Los derechos humanos como productos culturales: crítica del humanismo abstracto. Madrid: Catarata, 2005.

HOLANDA, Sérgio Buarque de. Raízes do Brasil. 26. ed. São Paulo: Companhia de letras, 2008.

Institut de Droit Internacional. L'asile en droit international public: à l'exclusion de l'asile neutre. Bath: Institut de Droit Internacional, 1950. Disponible sur : < http://www.idi-iil.org/idiF/navig_chron1933.html $>$. Acèss le : 31/07/2014.

International Court of Justice. Disponible sur : $<$ http://www.icj-cij.org/ pcij/series-a.php?p1=9\&p2=1>. Acèss le : 04/02/2014.

JELLINEK, Georg. Teoría general del Estado. Tradiccíon de Fernando de los Ríos. México: FCE, 2000.

. La Declaración de los Derechos del Hombre e del Ciudadano. Traducción de Adolfo Posada. México: Universidad Nacional Autónoma de México, 2000.

KELSEN, Hans. La unidad del derecho nacional y el derecho internacional (monismo e pluralismo). In: Teoría general del derecho y del Estado. 2. ed. 5 reimpresión. México: UNAM, p. 431-462, 1995.

KELSEN, Hans. Teoria pura do direito. 6. ed. Traduit par João Baptista Machado. São Paulo: Martins Fontes, 1999.

. Teoria geral do direito e do Estado. 3. ed. Traduit par Luís Carlos Borges. São Paulo: Martins Fontes, 2000.

LAFER, Celso. A internacionalização dos direitos humanos: Constituição, racismo e relações internacionais. São Paulo: Manole, 2005.

1982.

. Paradoxos e possibilidades. Rio de Janeiro: Nova Fronteira,

LEIVAS, Paulo Gilberto Cogo. Teoria dos direitos fundamentais sociais. Porto Alegre: Editora do Advogado, 2006.

MALBERG, Raymond Carré de. Contribuition à la Théorie general de l'État. Paris: Dalloz, 2004.

MARGUÉNAUD, Jean-Pierre. La Cour européene des droits del'homme. 3. ed. Paris: Dalloz, 2005. 
MBAYA, Etienne Richard. Relations between individual and collective human rights: the problema of rights of peoples. In: Law and State. vol. 46 , p. 7-23.

MENEZES, Wagner.A ONU e o Direito Internacional Contemporâneo. In: MEDEIROS, Antônio Paulo Cachapuz de (org.). Desafios do Direito Internacional Contemporâneo. Brasília: FUNAG, 2007, p. 325-342.

MORANGE, Jean. Droits de l'homme et libertés publiques. Paris: PUF, 1982.

NIETO-NAVIA, Rafael. International peremptory norms (jus cogens) and international humanitarian law. Disponible sur : <http://www. google.com.br/url? sa $=$ t\& $r c t=j \& q=\&$ esrc $=\mathrm{s} \& f r m=1 \&$ source $=$ web $\& c d=$ $4 \&$ ved $=0 \mathrm{CDoQFj} A D \&$ url $=\mathrm{http} \% 3 \mathrm{~A} \% 2 \mathrm{~F} \% 2 \mathrm{Fwww}$.iccnow.org $\% 2 \mathrm{Fdo}$ cuments\%2FWritingColombiaEng.pdf\&ei=RmvaUoXtPNLJkAek_oH AAQ\&usg=AFQjCNE7Resv5JyqU9cZkGA6MEfJG7ftwQ>. Acèss le: $17 / 01 / 2014$.

PARLETT, Kate. The individual in the international legal system: continuity and change in international law. Cambridge: Cambridge University Press, 2010.

PECES-BARBA, Gregorio. Derechos Fundamentales en la cultura jurídica española. Anuario de derechos humanos - 1981. Madrid: Instituto de Derechos Humanos de la Facultad de derecho, Universidad Complutense Madrid-3, 1981, p.169-253.

. La dignidad de la persona desde la filosofía del derecho. Madrid: Dykinson, 2003.

PÉREZ LUÑO, Antonio E. Temas claves de la Constitución Española: Los derechos fundamentales. 8. ed. Madrid: Tecnos, 2004.

- La universalidad de los derechos humanos y el Estado constitucional. Bogotá: Universidad Externado de Colombia, 2002.

PINHEIRO, Letícia. Política externa brasileira. Rio de Janeiro: Jorge Zahar Editor, 2004.

PORRÚA PÉREZ, Francisco. Teoría del Estado:Teoría política. Mexico: EdtorialParrúa, 1999.

RANGEL, Vicente Marotta. Evolução da Justiça Internacional. In: MEDEIROS, Antônio Paulo Cachapuz de (org.). Desafios do Direito Internacional Contemporâneo. Brasília: FUNAG, 2007, p. 79-100.

RAWLS, John. Direito dos povos. Traduit par Luís Carlos Borges. São Paulo: Martins Fontes, 2004.

ROBLEDO, Antonio Gómez. Le ius cogens internacional: sa gènese, sa nature, ses fonctions. Collected Courses of The Hague Academy of 
internacional law, 1981, p. 9-218.

ROSS, Alf. Direito e Justiça. Traduit par Edson Bini. Bauru: Edipro, 2003.

SALOMÃO LEITE, George; SALOMÃO LEITE, Glauco. A abertura da constituição em face dos princípios constitucionais. In: SALOMÃO LEITE, George. Dos princípios constitucionais: considerações em torno das normas principiológicas da Constituição. São Paulo: Malheiros, 2003, p. 136-164.

SCHWARTZENBERG, Roger-Gérard. Sociologia política: elemento de ciência política. Traduit par Domingos Mascarenhas. São Paulo: Difel, 1979.

SORTO, Fredys Orlando. Considerações sobre a primeira Corte internacional de Justiça. In: Solução e prevenção de litígios internacionais. São Paulo: NECIN-CAPES; Porto Alegre: Livraria do Advogado, p. 275-316, 1999.

. A Doutrina Bush das guerras preventivas e o Sistema das Nações Unidas. In: MERCADANTE, Araminta de Azevedo; MAGALHÃES, José Carlos (Orgs.). Reflexões sobre os 60 anos da ONU. Ijuí: Unijuí, 2005, p. 130-163.

. A Declaração Universal dos Direitos Humanos no sexagésimo aniversário. Verba Juris: Anuário de Pós-Graduação em Direito, João Pessoa, ano 7, n. 7, p. 9-34, jan./dez. 2008.

. O projeto jurídico de cidadania universal: reflexões à luz do direito de liberdade. Anuário hispano-luso-americano de derecho internacional, Madrid, vol. 20, p. 103-126, ene./dic. 2011.

- A condição da pessoa humana no Projeto de Código de Direito Internacional Público de Epitácio Pessoa. In: Epitácio Pessoa e a codificação do direito internacional. Porto Alegre: Sergio Antonio Fabris, 2013, p. 133-155.

STRAUSS, Leo. Droit naturel et histoire. Paris: Flammarion, 1986, p. 99.

. La ciudad y el hombre. Buenos Aires: Katz, 2006.

SUDRE, Frédéric. Droit européen et international des droits de l'homme. 11. ed. Paris: Presses Universitaires de France, 2012.

TONNIES, Fernand. Comunidade e sociedade como entidades típicoideais. In: FERNANDES, Florestan (org.). Comunidade e sociedade: leituras sobre problemas conceituais, metodológicos e de aplicação. São Paulo: Editora da Universidade de São Paulo, [S.d].

TRIEPEL, Karl Heinrich. As relações entre o direito interno e o 
internacional. Revista da Faculdade de Direito. Belo Horizonte: UFMG, ano 17, n. 6, p. 7-64, 1966.

TRINDADE, Antônio Augusto Cançado. Princípios do direito internacional contemporâneo. Brasília: Universidade de Brasília, 1981.

. Desafios para a Efetiva Proteção Internacional dos Direitos Humanos. In: MEDEIROS, Antônio Paulo Cachapuz de (org.). Desafios do Direito Internacional Contemporâneo. Brasília: FUNAG, 2007, p. 207-321.

A consolidação da capacidade processual dos indivíduos na evolução da proteção internacional dos direitos humanos: quadro atual e perspectivas na passagem do século. Seminário Direitos Humanos no Século XXI, 10 e 11 de setembro de 1998, Instituto de Pesquisa de Relações Internacionais, Fundação Alexandre Gusmão, Rio de Janeiro. Disponible sur : $<$ http//:www.mre.gov.br/ipri> Acèss le : 07/052013.

. The legal personality of individual as subject of international law. In: International Law for Humankind: Towards a New Jus Gentium. Disponible sur : <http://books.google.com.br/books?id=5a8 h4BPfkZ4C\&pg=PR8\&lpg=PR8\&dq=Trindade+The+legal + personali ty + of + individual + as + subject + of + international + law. \& source $=$ bl\&ots $=$ GJxn5rxBez\&sig=pIz6D7szoPt1YD6H3KMQkOJmmUQ\&hl=pt- BR $\& s a=X \&$ ei $=y 13 \mathrm{dUvmGMeTNsQSH9oLACQ \& ved=0CCoQ6AEwA}$ $\mathrm{DgK} \# \mathrm{v}=$ onepage $\& \mathrm{q}=$ Trindade $\% 20 \mathrm{The} \% 20$ legal $\% 20$ personality $\% 20$ of $\% 20$ individual $\% 20$ as $\% 20$ subject $\% 20$ of $\% 20$ international $\% 20$ law.\&f=false $>$. Acèss le: $20 / 01 / 2017$.

TRINDADE, Antônio Augusto Cançado; PEYTRIGNET, Gérard; RUIZ DE SANTIAGO, Jaime; INSTITUTO INTERAMERICANO DE DIREITOS HUMANOS; COMITÊ INTERNACIONAL DA CRUZ VERMELHA; ALTO COMISSARIADO DAS NAÇÕES UNIDAS PARA REFUGIADOS. As Três Vertentes da Proteção Internacional dos Direitos da Pessoa Humana: Direitos Humanos, Direito Humanitário e Direito dos Refugiados. San José; Brasília: ACNUR: CICV: IIDH, 1996.

VALLADÃO, Haroldo. Democratização e socialização do Direito Internacional: Os impactos latino-americanos e afri-asiáticos. Rio de Janeiro: Livraria José Olympio, 1961.

VALLADARES, Gabriel Pablo. Apuntes sobre algunos tópicos e institutos de relevancia jurídica para la implementación a nivel nacional de las violaciones a las normas del derecho internacional humanitario. In: Anuário Brasileiro de Direito Internacional, vol. I. Belo Horizonte: Editora CEDIN, 2007. 